\title{
Research Paper \\ Toxicity and Teratogenic Effects of Zinc Sulfide Nanoparticles on Chick Embryo and Chick Fibroblast Cell Culture
}

\author{
*Asadollah Asadi ${ }^{1}$, , Arash Abdolmaleki ${ }^{2,3}$ \\ 1. Department of Biology \& Geology, Faculty of Science, University of Mohaghegh Ardabili, Ardabil, Iran. \\ 2. Department of Engineering Sciences, Faculty of Advanced Technologies, University of Mohaghegh Ardabili, Namin, Iran \\ 3. Department of Engineering, Faculty of Advanced Technologies, Sabalan University of Advanced Technologies, Namin, Iran.
}

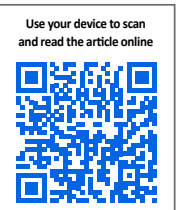

Citation Asadi A, Abdolmaleki A. [Evaluation of Toxic and Teratogenic Effects of Zinc Sulfide Nanoparticles on Chicken Embryos and Fibroblastic Cells Culture (Persian)]. Quarterly of "The Horizon of Medical Sciences". 2019; 25(4):270-281. https://doi. org/10.32598/hms.25.4.270

https://doi.org/10.32598/hms.25.4.270

Key words:

Nanoparticles, Toxic-

ity, Malformation,

Chick embryo, Zinc

sulfide

\section{ABSTRACT}

Aims Nanoparticles (particles with a diameter of $10-500 \mathrm{~nm}$ ) are currently used in the cosmetics industry as well as for pharmaceuticals, diagnostic imaging, and tissue engineering. Since these nanoparticles are used in industry and drug delivery, they can also be used by pregnant women. Thus, the current study investigated the teratogenic and cytotoxic effects of Zinc Sulfide ( $\mathrm{ZnS}$ ) nanoparticles on the embryo and their fibroblastic cell culture.

Methods \& Materials Zinc sulfide ( $\mathrm{nnS}$ ) nanoparticles were synthesized. Then, nanoparticles at the concentrations of 5, 10,15,30, and $40 \mathrm{mg} / \mathrm{mL} / \mathrm{egg}$ were injected into the air sac of the eggs in three replicates on the third day of incubation. Next, the treated and control eggs, on day 19 of incubation were opened, and embryos were weighted, and the relevant mortality rate was recorded. Fibroblast cells were isolated, cultured, and treated from the control embryo, and morphological changes and cell survival percentages were recorded.

Findings The obtained results revealed that the embryos' survival rate depends on the nanoparticle concentration. As a result, at the highest concentration, only $36.32 \%$ of the embryos survived, and the lethal dose $50 \%$ (LD50) was equal to $32.47 \mathrm{mg} / \mathrm{egg}$. Morphological study of the treated embryos club foot and skeletal staining suggested the deletion of caudal vertebrate. The cytotoxicity study results of ZnS nanoparticles on fibroblastic cells indicated the survival fractions of $88.45 \%, 68.75 \%$, and $49.32 \%$, respectively, and its IC50 value was measured aas1460 $\mu \mathrm{M}$.

Conclusion The present study results suggested that ZnS nanoparticles had no significant toxic effects on the embryos and culture of chicken fibroblastic cells at low concentrations.

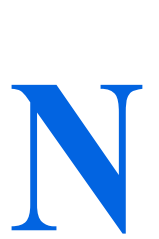

Extended Abstract

\section{Introduction}

anoparticles (particles with a diameter of $10-500 \mathrm{~nm}$ ) are currently used in the cosmetics industry as well as for pharma- these nanoparticles are used in industry and drug delivery, they can also be used by pregnant women. Thus, the current study investigated the teratogenic and cytotoxic effects of Zinc Sulfide $(\mathrm{ZnS})$ nanoparticles on the embryo and their fibroblastic cell culture.

ceuticals, diagnostic imaging, and tissue engineering. Since

\section{* Corresponding Author:}

\section{Asadollah Asadi, PhD.}

Address: Department of Biology \& Geology, Faculty of Science, University of Mohaghegh Ardabili, Ardabil, Iran

Tel: +98 (45) 31505187

E-mail: asad.asady@gmail.com 


\section{Methods}

Asadi et al. evaluated the effects of nano polymer BDP18, as a carrier with slow drug-release property used in cancer treatment on chick embryos. Their results indicated that BDP18 has low toxic and teratogenic effects only at high doses; therefore, it can be used at low concentrations as an effective drug release system [1]. In another study, the toxic and physiological effects of platinum nanoparticles widely used in the treatments were estimated on chick embryo development. The relevant results reflected that these nanoparticles induce apoptosis in the brain tissues [2].

Ninety Ross hatching eggs were obtained from a local company (Arta Jujeh). The research was carried out in the research laboratory of the Biology Department of Mohaghegh Ardabili University in Summer 2018.

\section{Sampling}

In this study, a random sampling method was used. Moreover, for each concentration, on average, 3 groups of 5 samples were selected.

\section{Tools}

Alizarin red staining, culture medium, Dimethyl Sulfoxide (DMSO), Multi-and Table Tournament (MTT) colorimetric assay were used in the present research.

\section{Results}

The morphological examination of embryos suggested low abnormalities in the treated specimens; no abnormality was observed up to the concentration of $30 \mathrm{mg} / \mathrm{mL}$ per egg. The assessment of the relationship between treatment concentration and frequency of survived embryos revealed a lethal dose $50 \%$ (LD50) of $32.47 \mathrm{mg} / \mathrm{egg}$. The results of the effect of 500, 1000, and $1500 \mu \mathrm{M} \mathrm{ZnS}$ concentrations on chick embryo fibroblastic cells after 16 hours reported the survival fractions of $88.45 \%, 68.75 \%$, and $49.32 \%$ respectively. Therefore, it can inhibit cell growth with an LC50 of $1460 \mu \mathrm{M}$.

\section{Discussion}

Studies have indicated that embryonic mortality after injection into the egg can be due to the destruction and disruption of embryonic homeostasis, as well as the susceptibility of embryos to their developmental stage [3]. Many researchers have demonstrated the teratogenic effect of antibiotics and growth factors when injected into the egg during the first week of pregnancy [4]. Various nanopar- ticles have been generated and used as contrast agents in diagnosis and imaging, as well as drug delivery [5].

The effect of $\mathrm{ZnS}$ nanoparticles on chick embryos resulted in the skeletal malformations of the caudate vertebrae. This may be due to its direct effect on the loss of embryonic cells growing in the caudate vertebra. In this regard, evaluating the effect of methotrexate in rats suggested that most of the abnormalities in rats were due to the injections of methotrexate confined to caudal vertebrae [6]. Previous studies have indicated that the bones of the lower extremities are more prone to be affected by teratogenic drugs [7]. Barnes et al. (1996) argued that valproic acid reduces the transcription rate of Pax1 in chicken somites [8].

Studnicka et al. examined the effect of nanoparticles of silver/palladium alloy on chicken embryo development; they concluded that these nanoparticles do not affect embryo growth and weight [9]. Bavanilatha and Stanley assessed the effect of silver nanoparticles on chicken embryo. Heart, eye, and liver tissues were examined after chickens were injected on day 18 of incubation. Their results reflected no changes in heart, eye, and liver tissue cells [10]. In another study, teratogenic and cytotoxic effects of salen-vanadium complex on chicken embryos, hepatic, and fibroblastic-cell cultures were evaluated. According to their results, at high concentrations in the culture medium, fibroblastic cells became dense, and their interconnections were loosed [28].

\section{Recommendations}

It is recommended to investigate the chemical properties of nanoparticles and provide further information in this respect.

\section{Limitations}

There were no limitations to the current study.

\section{Conclusion}

The threshold effect of $\mathrm{ZnS}$ nanoparticles in this study was $32.47 \mathrm{mg} / \mathrm{mL}$ per egg. It had no significant effects on the chick embryo at lower doses and no morphological and skeletal teratogenic effects at doses lower than the threshold value. Therefore, when using $\mathrm{ZnS}$ in various industries, such as pharmacy, cosmetics production, and other related industries, its threshold level of toxicity and malformations for embryo should be considered. 


\section{Ethical Considerations}

Compliance with ethical guidelines

All animal experiments were carried out in accordance with local University of Mohaghegh Ardabili (UMA) committee for Human and Animal ethics.

Funding

University of Mohaghegh Ardabili support the financial of this study.

Authors' contributions

Participated in research design: Asadollah Asadi, Conducted experiments: Arash Abdolmaleki; Performed data analysis, wrote or contributed to the writing of the manuscript: All authors.

Conflicts of interest

The authors declare no conflicts of interest.

Acknowledgements

The authors would like to thank the Research Council of University of Mohaghegh Ardabili for the financial support of this study. 


\title{
بررسى اثرات سميت و تراتوزنيك نانوذرات سولفيد روى بر جنين جوجه و كشت سلولهاى فيبروبلاستى جوجه
}

\author{
"اسداله اسدى' • م، آرش عبدالملكى" \\ I. كروه زيستشناسى، دانشكده علوم، دانشكاه محقق اردبيلى، اردبيل، ايران.

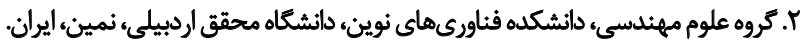

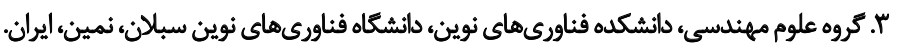

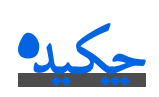

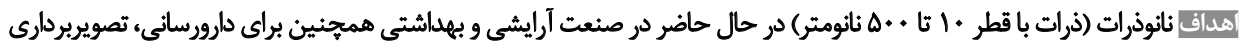

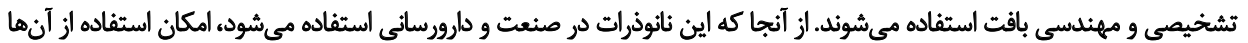

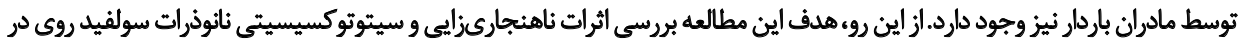

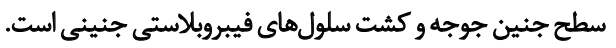

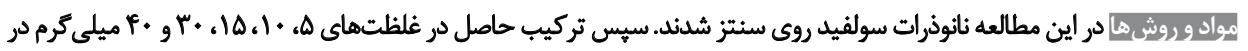

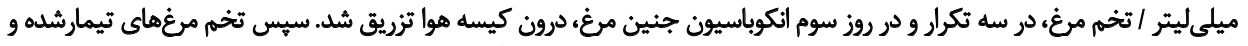

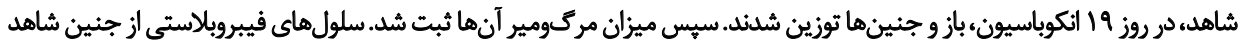

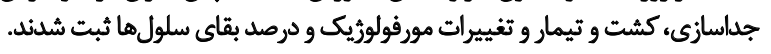

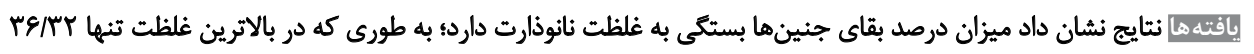
درصد از جنينها زنده ماندند و ميزان(LD

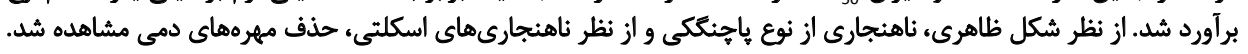

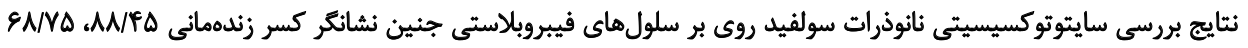

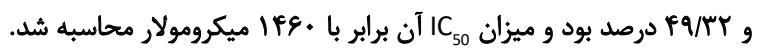

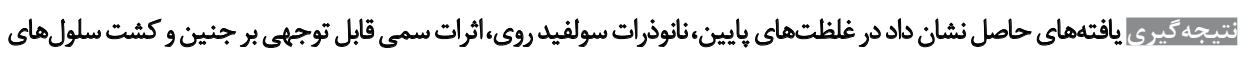

تاريخ دريافت: V• بهمن VIrav تاريخ يذيرش: الم مرداد

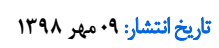
فيبروبلاستى جوجه ندارد.

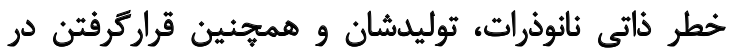

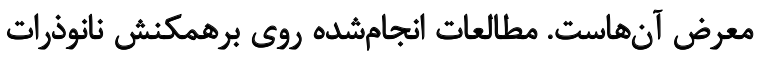

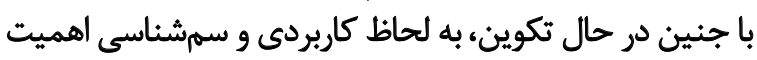

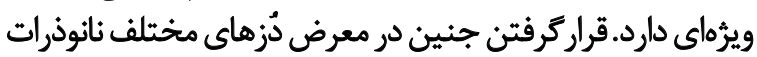

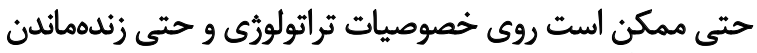

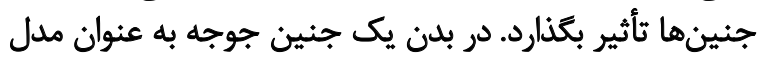

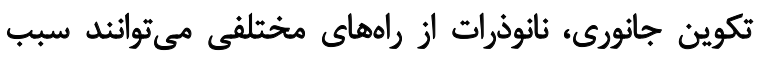

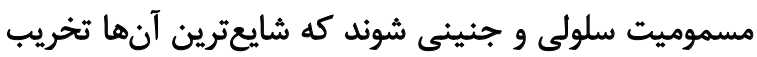

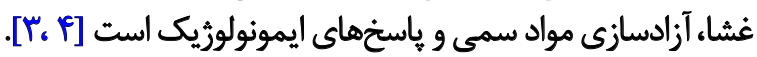
نانوذرات در حال حاضر از طيف وسيعى از مواد ساخته

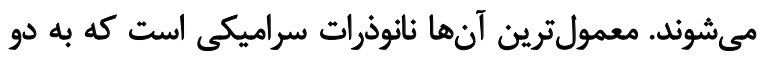

حوزه فناورى نانو بهسرعت در حال رشد است و تخمين

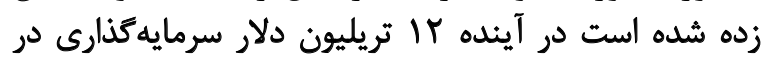

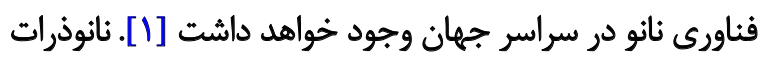

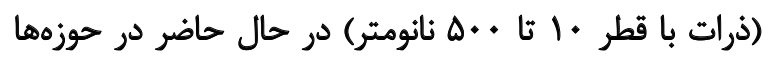

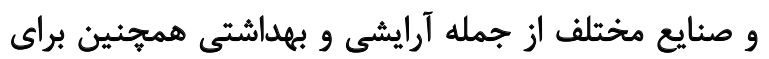

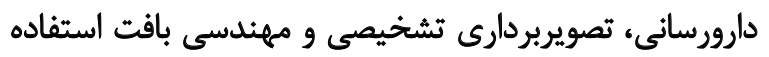

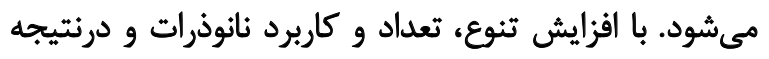

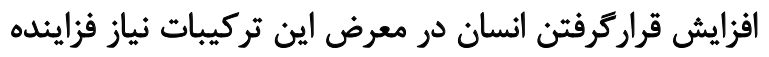

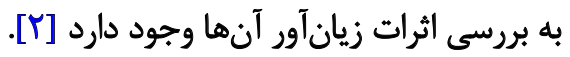


تكوين اسكلت جنين جوجه نهتنها در مطالعات جنينشناسى

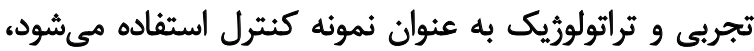

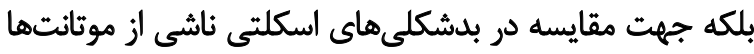

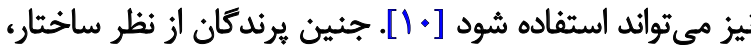

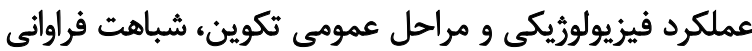

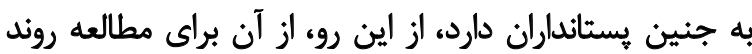

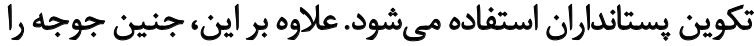

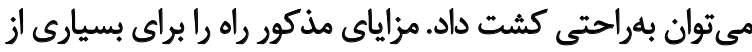

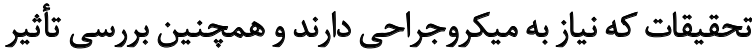

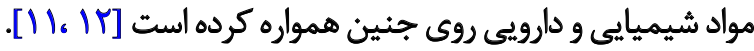
در مورد اثرات نانوذرات بر سلولها و جنين، اطلاعات بسيار

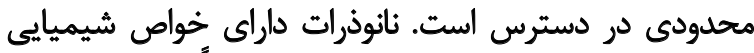

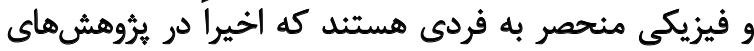

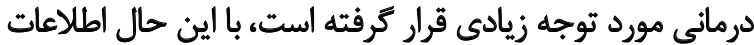

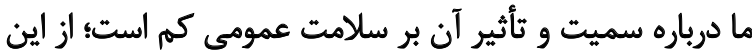

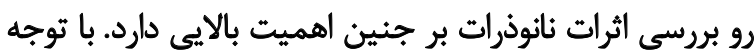

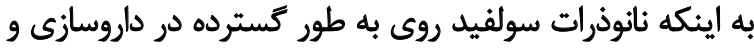

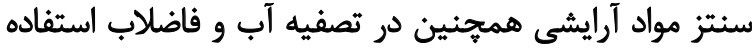

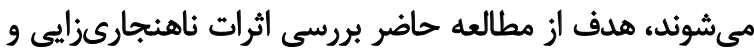

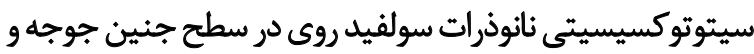
كشت سلول هاى فيبروبلاستى جنينى است.

\section{مواد وروشها}

يثروهش حاضر از نوع تجربى است كه در آزمايشعاه تحقيقاتى

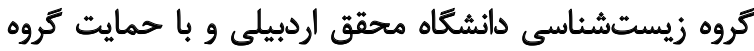
زيستشناسى دانشكاه محقق اردبيلى انجام شده است.

\section{تبهيه نائوذورات سولثيد روى با استثادها أو امواج ريزموج}

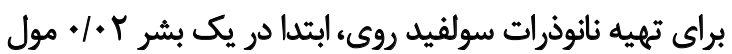

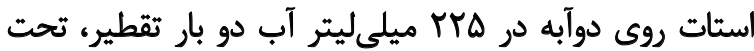

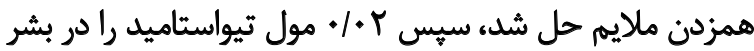

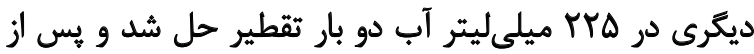

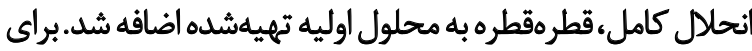

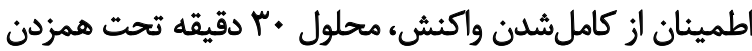

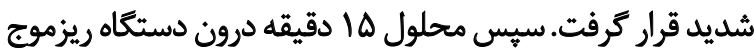

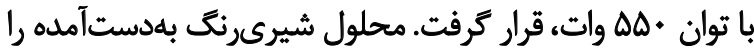

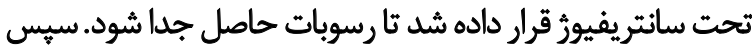

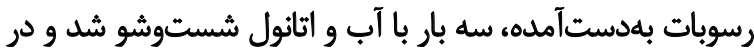

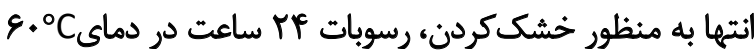

در داخل آون قرار داده شد [بآ].

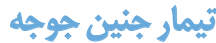

9 تخم من بارور نراد راس از شركت محلى (آرتا جوجه)
بخش سراميكهاى اكسيد فلزى نظيراكسيدهاى تيتانيوم، روى،

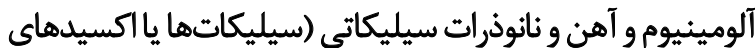

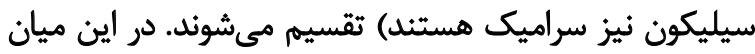
نانوذرات روى در مصارف يزشكي، دارئ داروسازى و آرايشى استفاده

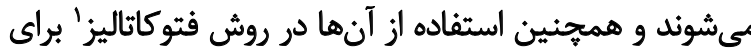

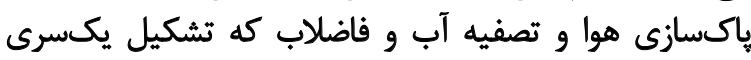

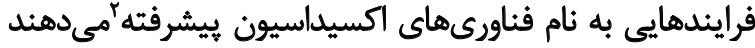
بيشتر از همه مورد توجه قرار كرفته است [هائه

از ديدكاه سمشناسى عوامل مختلفى در موجودات زينده به

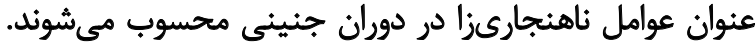

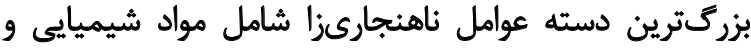

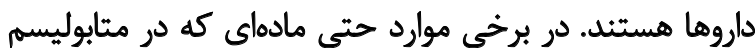

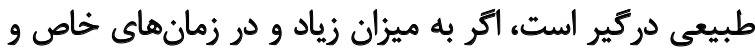

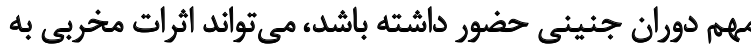

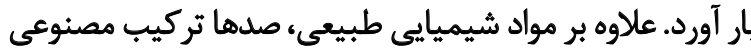

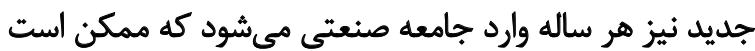

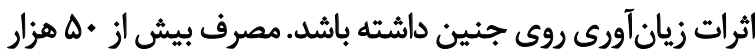

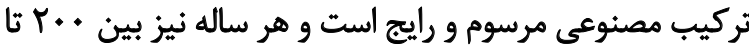

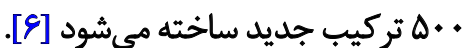

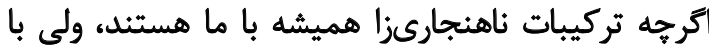

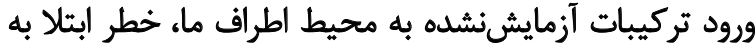

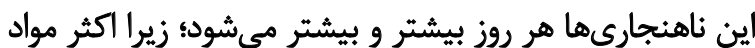

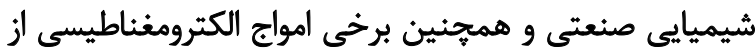

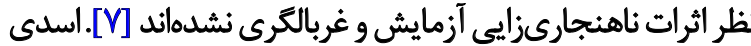

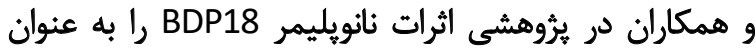

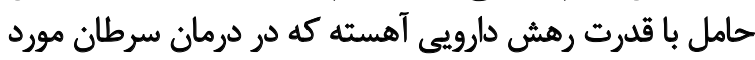

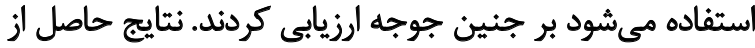

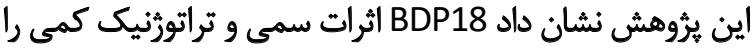

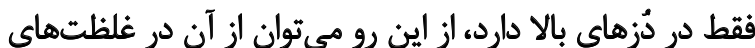

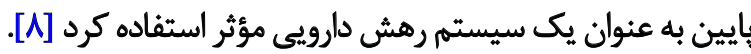
در يروهش ديكرى اثرات سميت و فيزيولوزيك نانوذرات

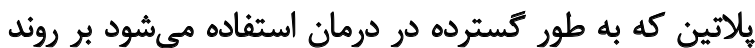

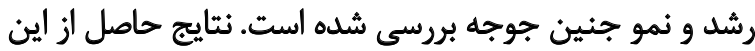
عرؤش نشان داد اين نانوذرات سبب القاى آيويتوز در سلولهاي

عصبى ميى شود [9].

جنين جوجه يكى از مدل هاى آزمايشكاهى جانورى مناسب

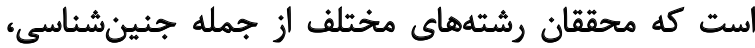
فارماكولوزى، فيزيولوزى و وبيوشيمى از آن آن استفاده مي كنيند.

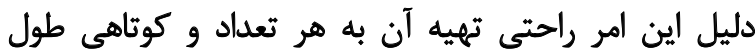

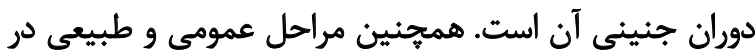

1. Photocatalysis

2. Advanced Oxidation Technologies (AOTs) 
شدند. شفافسازى نهايى با قراردادن جنين در كُليسرول

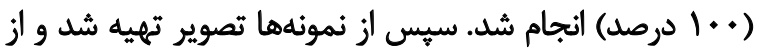

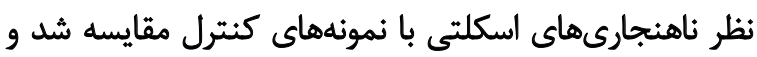

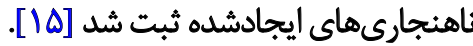

\section{جيدأسازي و كشت سلولهاي فيبروبالاستى جوجها.}

جهت جداسازى سلولهاى فيبروبلاستى، جنينها در روز

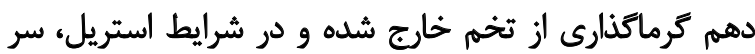

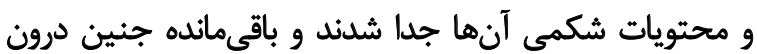

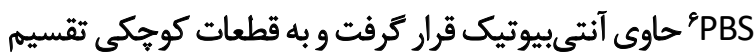

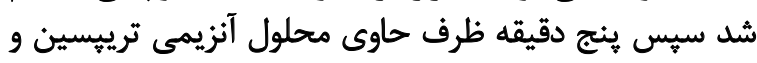

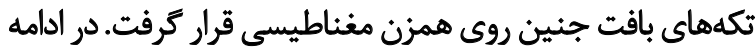

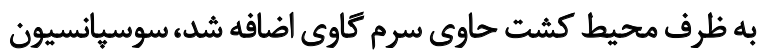

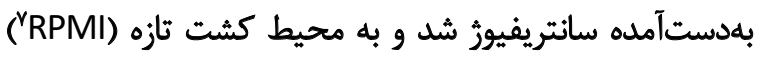

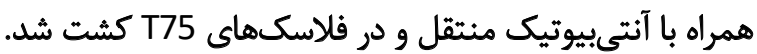

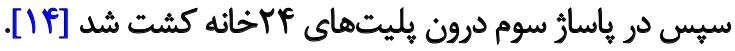

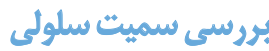

براى بررسى ائرات سايتوتوكسيسيتى نانوذره سولفيد روى، از بازي

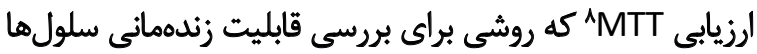

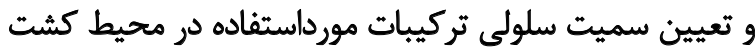

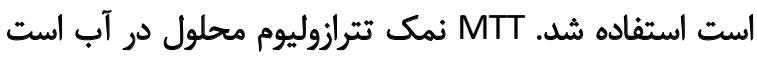

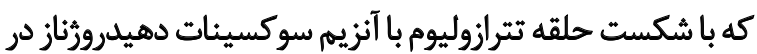

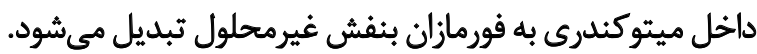

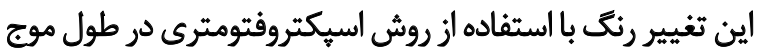
هf.

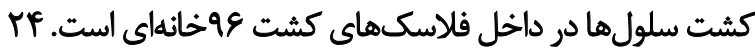

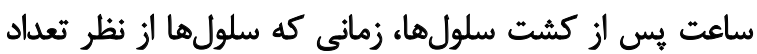

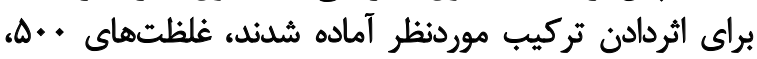

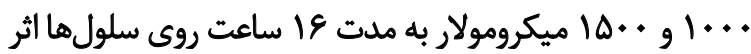

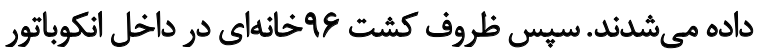

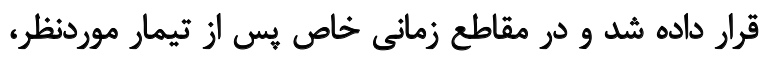

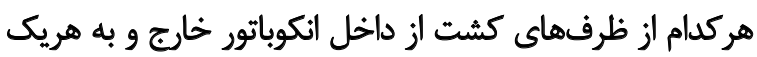

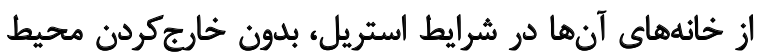

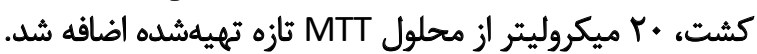

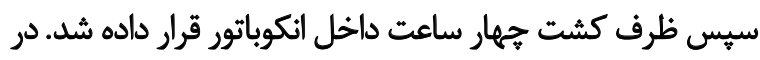

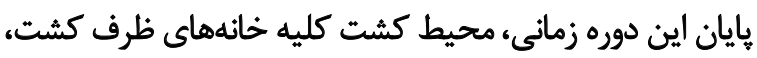

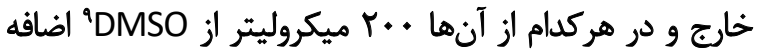

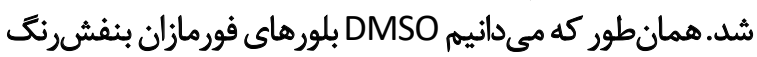

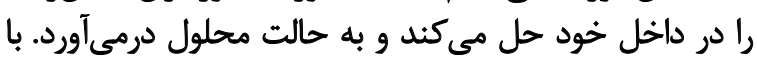

6. Phosphate buffered saline

7. Roswell park memorial institute medium

8. (4,5-dimethylthiazol-2-YI)-2,5-diphenyltetrazolium bromide 9. Dimethylsulfoxide

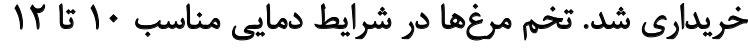

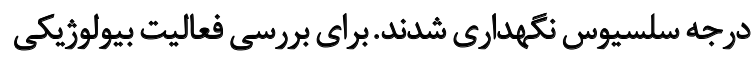

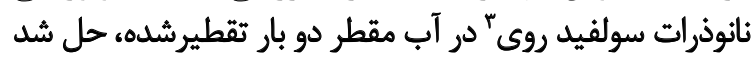

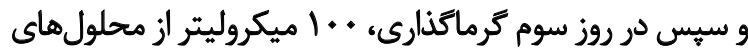

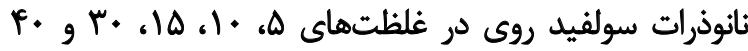

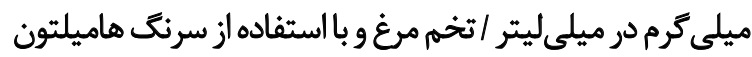

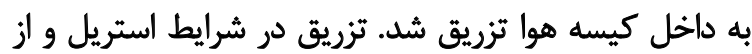

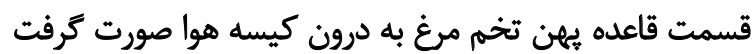

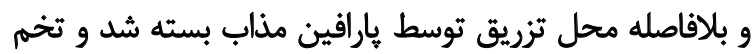

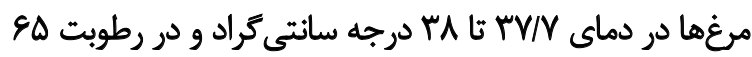

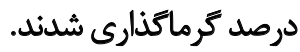

به عنوان شاهد از تعداد تكرار يكسان تخم مرغ باتئريق

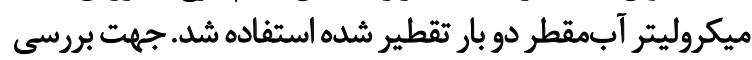

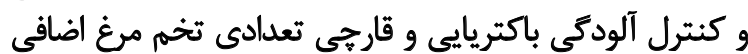

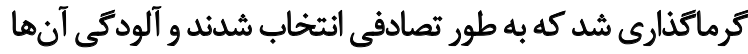

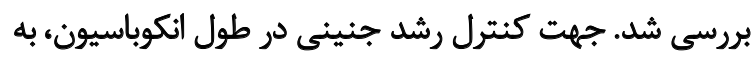

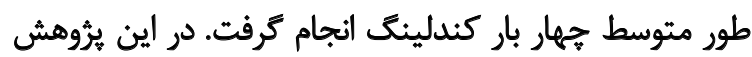
براى هر غلظت به طور متوسط سه كروه ينجنايى انتخاب شد

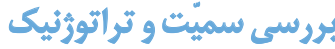

تخم مغٔهاى تيمارشده و شاهد، در روز 19 انكوباسيون، باز

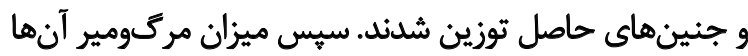

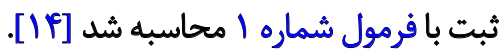

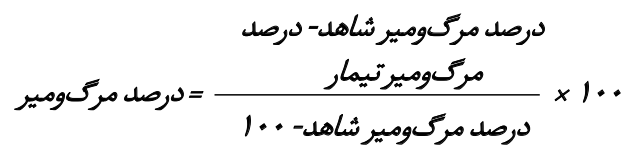

به منظور بررسى ناهنجارىهاى مورفولوزيكى، جئينها

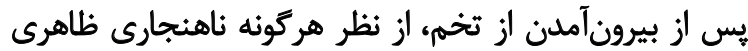

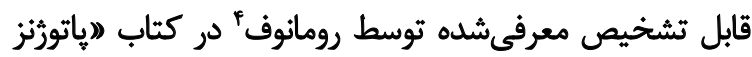

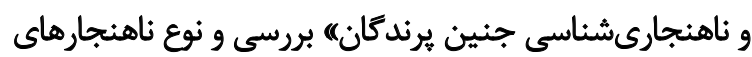

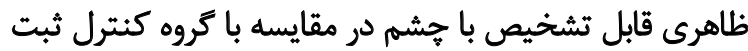

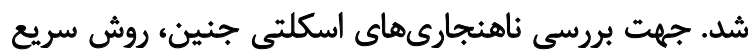

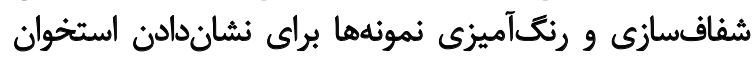

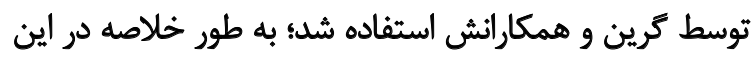

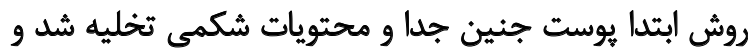

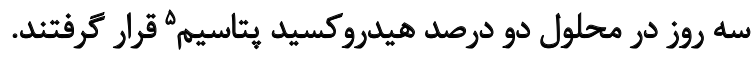

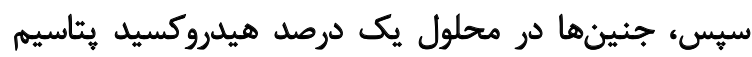

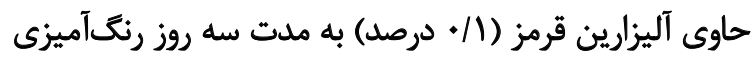

3. Zinc Sulfide (ZnS)

4. Romanoff

5. Potassium Hydroxide (KOH) 
بود. به عبارتى ديكر، افزايش مركَومير نسبت به افزرايش غلظت

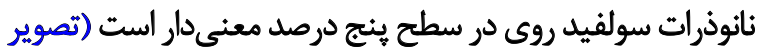

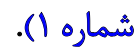

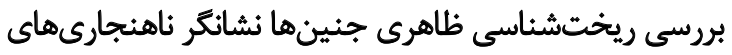

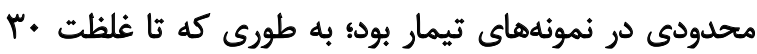

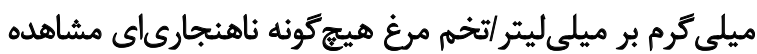

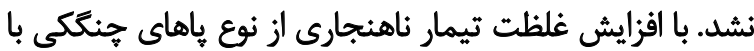

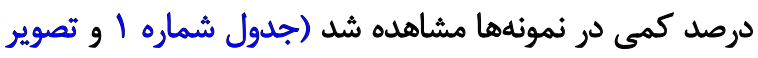

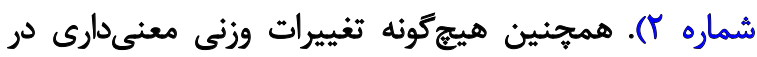
جنينهاى تيمارشده با نانوذرات سولفيد روى در مقايسه با كروه شم مشاهله نشد.

بررسى ساختار اسكلتى جنينهاي تحت تيمار نشان داد تا

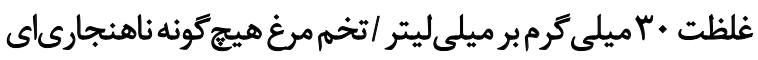

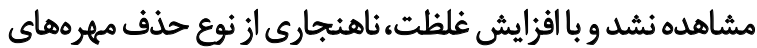
دمى مشاهده شد (جدول شماره ا و تصوير شماره بار).

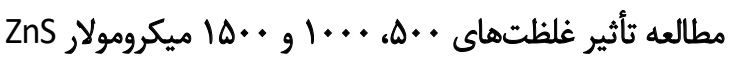

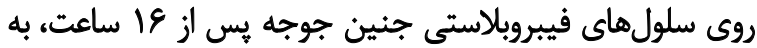

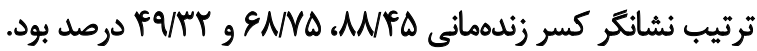

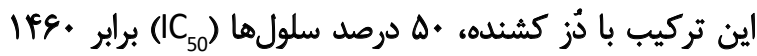

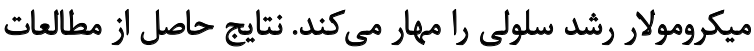

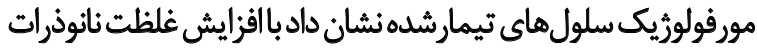

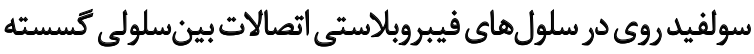

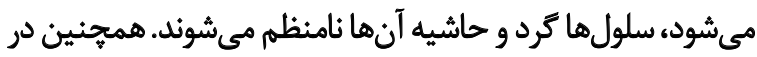

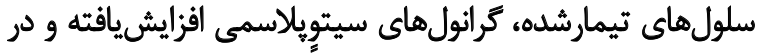

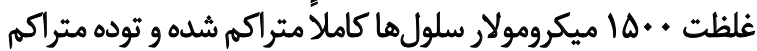
سلولى بلهتريج كسسته مي شود (تصوير شماره f).
حلشدن كامل بلورها و ايجاد محلول هاى يكنواخت، جذب نورى هركدام از خانهها با استفاده از دستكاه

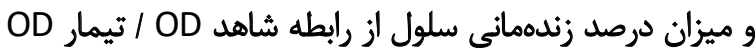

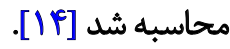

\section{تُحليل آمارى}

طرح آزمايش از نوع طرح كاملاً تصادفى است. براى تحليل

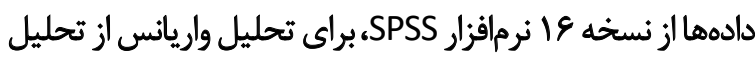

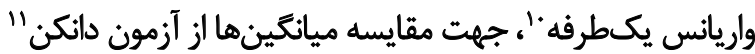

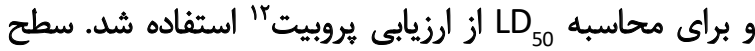

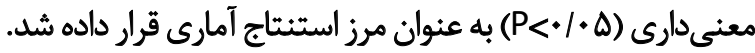

يافتهها

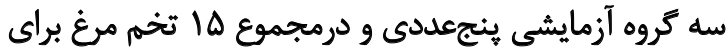

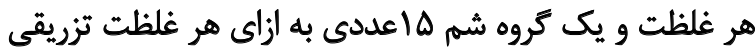

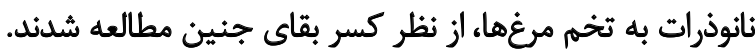

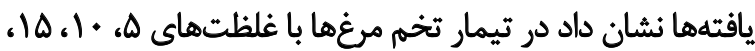

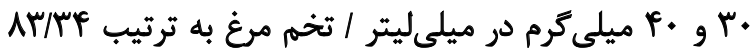

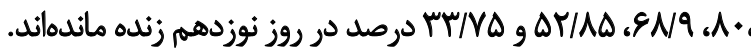

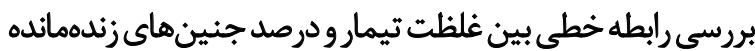

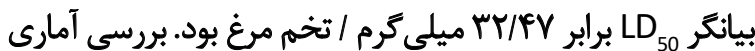

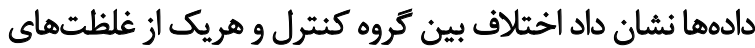

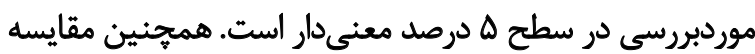

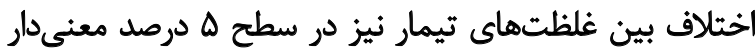

\section{ANOVA}

11. Duncan

12. Probit

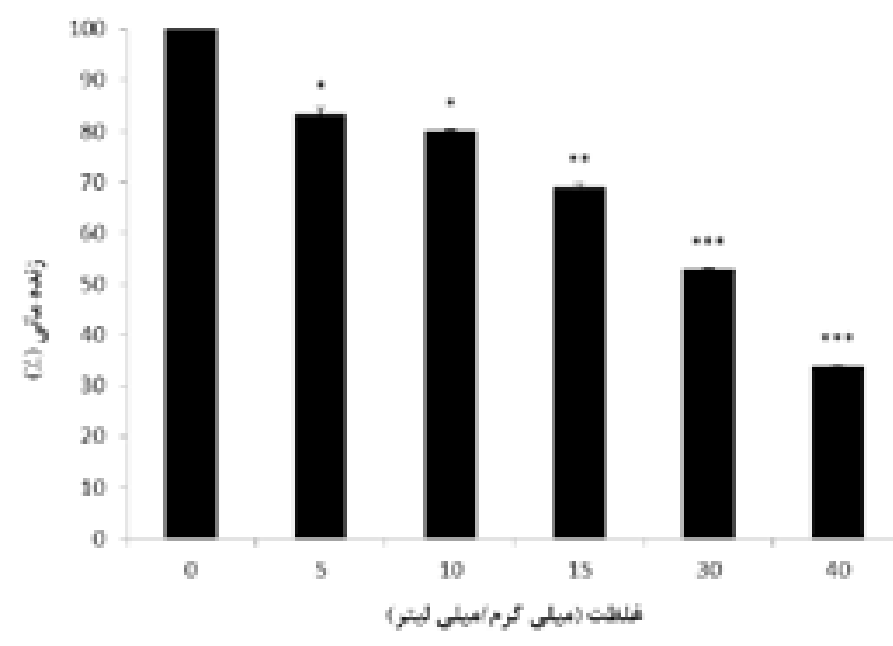

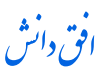

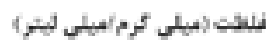

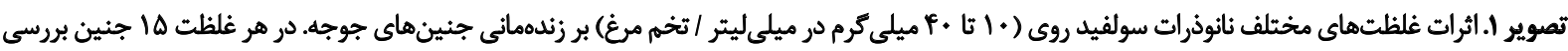

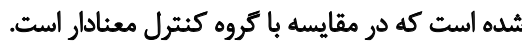
$* * * P<\cdot 1 \cdot .1, * * P<\cdot 1 \cdot 1, * P<* 1 \cdot \Delta$ 
جدول ا. ناهنجارىهاى مربوط به غلظتهاى مختلف نانوذرات سولفيد روى، بر جنين جوجه

\begin{tabular}{|c|c|c|c|c|}
\hline نوع ناهنجارى & ورصد ناهنجارى اسكلتى & نوع ناهنجارى & مورفد ناهنجارىى & ميكرومولار / تخلخم هرغ \\
\hline- & تشخيص داده نشد & - & تشخيص داده نشل & • \\
\hline- & تشخيص داده نشّد & - & تشخيص داده نشد & $\Delta$ \\
\hline- & تشخيص داده نشد & - & تشخيص داده نشد & 1. \\
\hline- & تشخخيص داده نشُ & - & تشخخيص داده نشلد & 10 \\
\hline حذف مهرمهاى دمى & e/ro & ياهاي جنككى & $\Delta / M$ & r. \\
\hline حذف مهرههاي دمى & $\Delta / M$ & باهاى جنككى & $1 \% / \pi r$ & f. \\
\hline
\end{tabular}

تخريب و بههمخوردن تعادل هوموستازى جنينى به علت تزريق

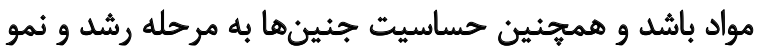

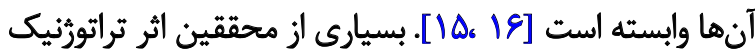

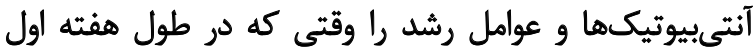

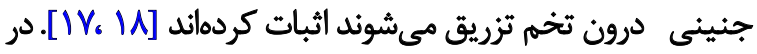

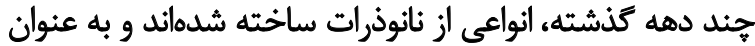

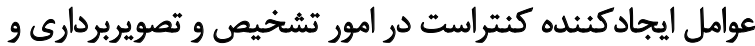

دارورسانى استفاده شدهاند [19].

مطالعه حاضر نشان داد تزريق نانوذرات سولفيد روى در روز

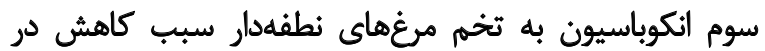

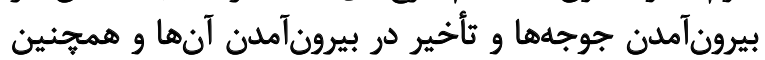

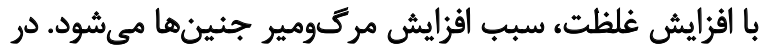

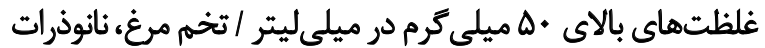

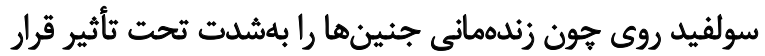

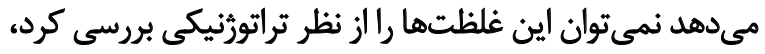
ثأثير تركيب نانوذرات سولفيد روى بر جنين جوجه به به ايجاد

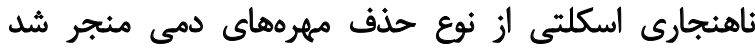

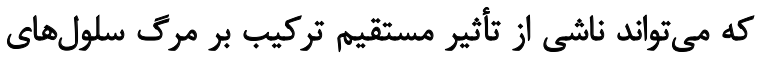

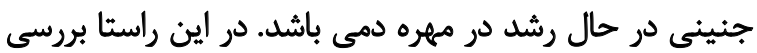

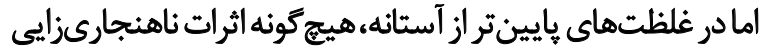

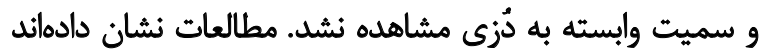

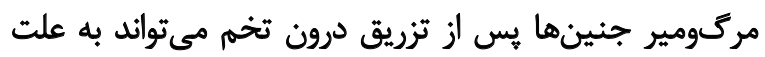
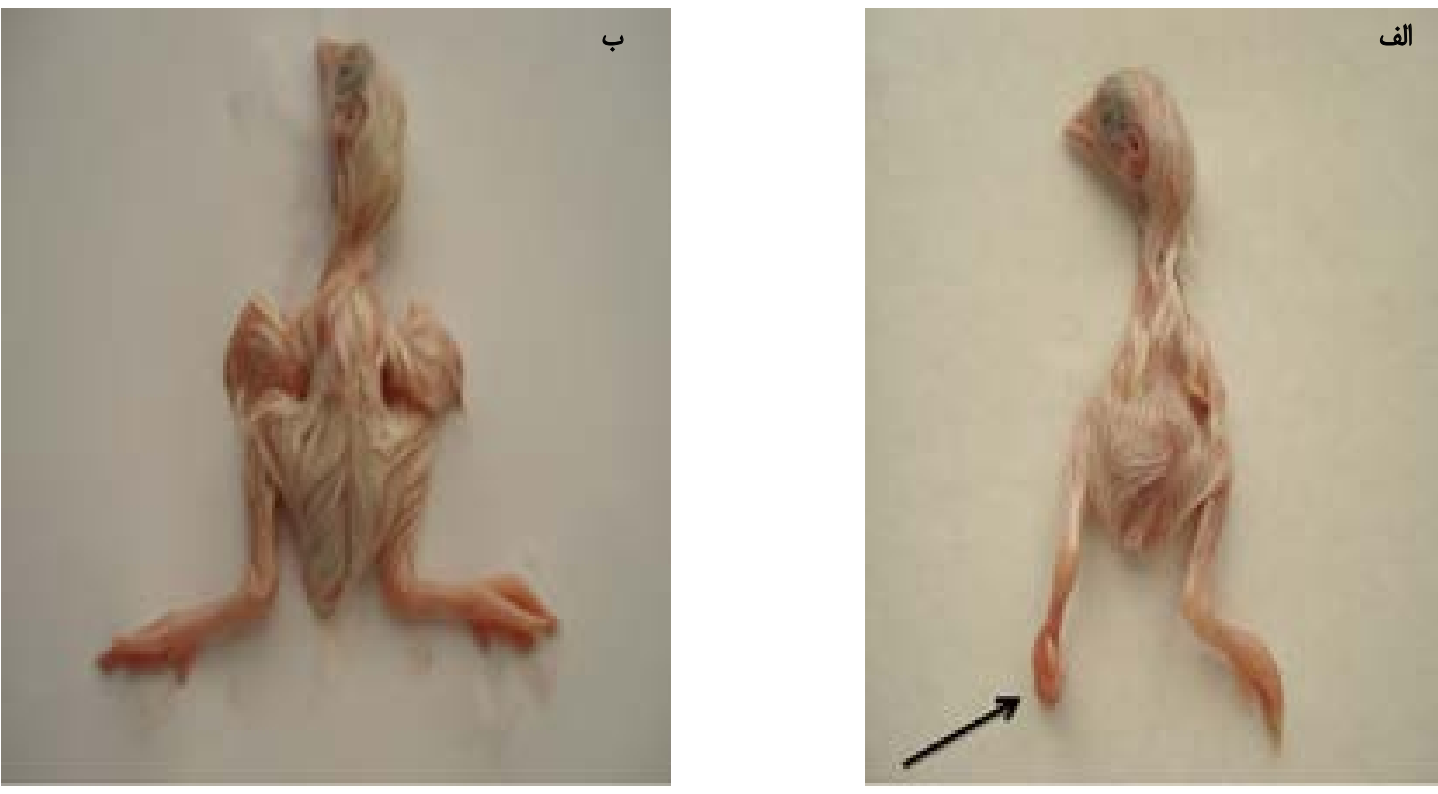


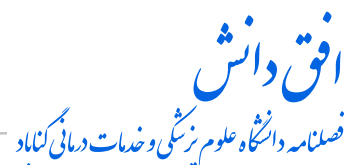
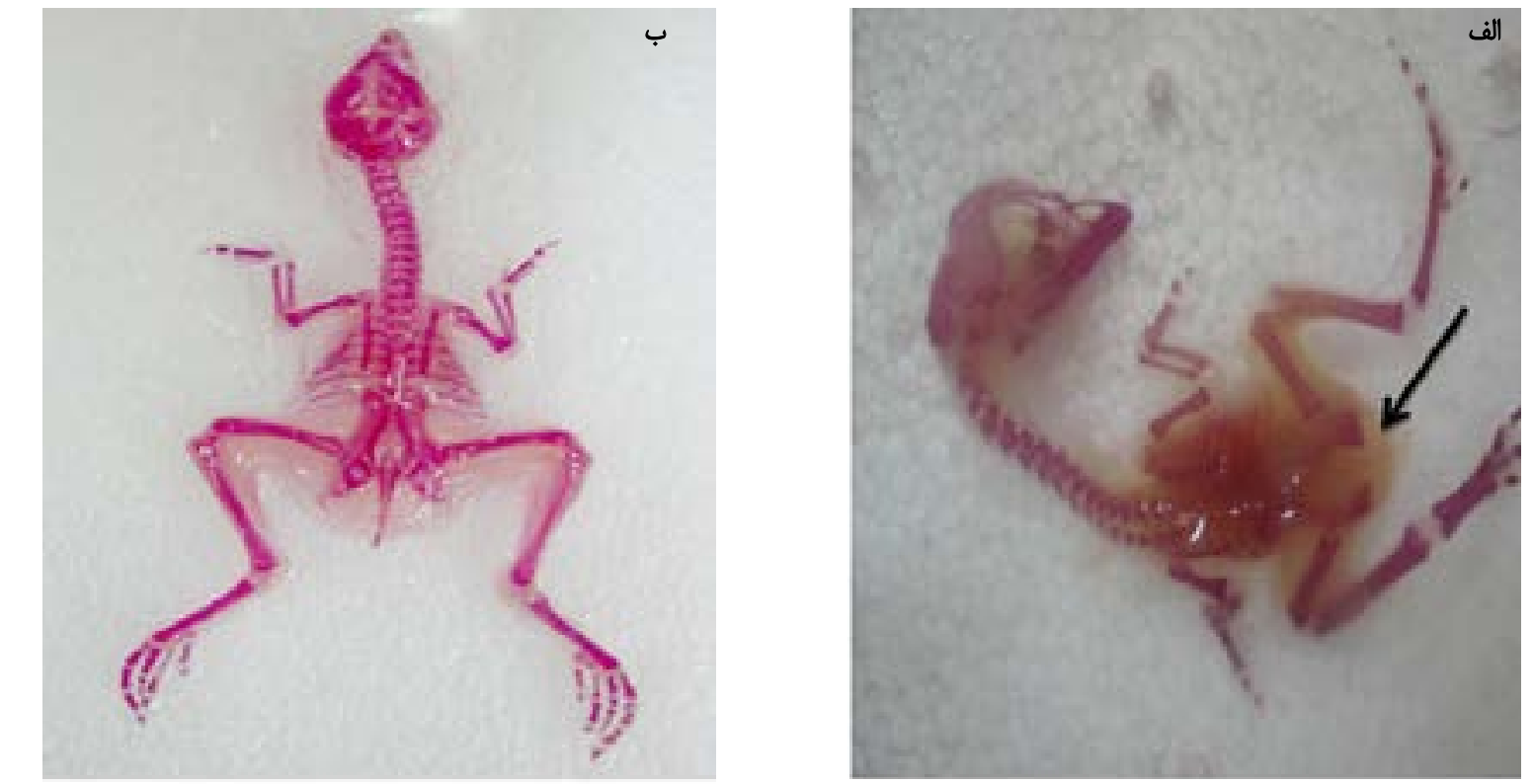

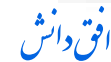

تصوير ب. ناهنجارى اسكلتي الف: ناشى از غلظت •f ميلى كرم در ميلى ليتر / تخم مرغ به صورت حذف مهرهماى دمى؛ ب: در مقايسه با نمونه جوجه سالم حاصل از جنين شاهد
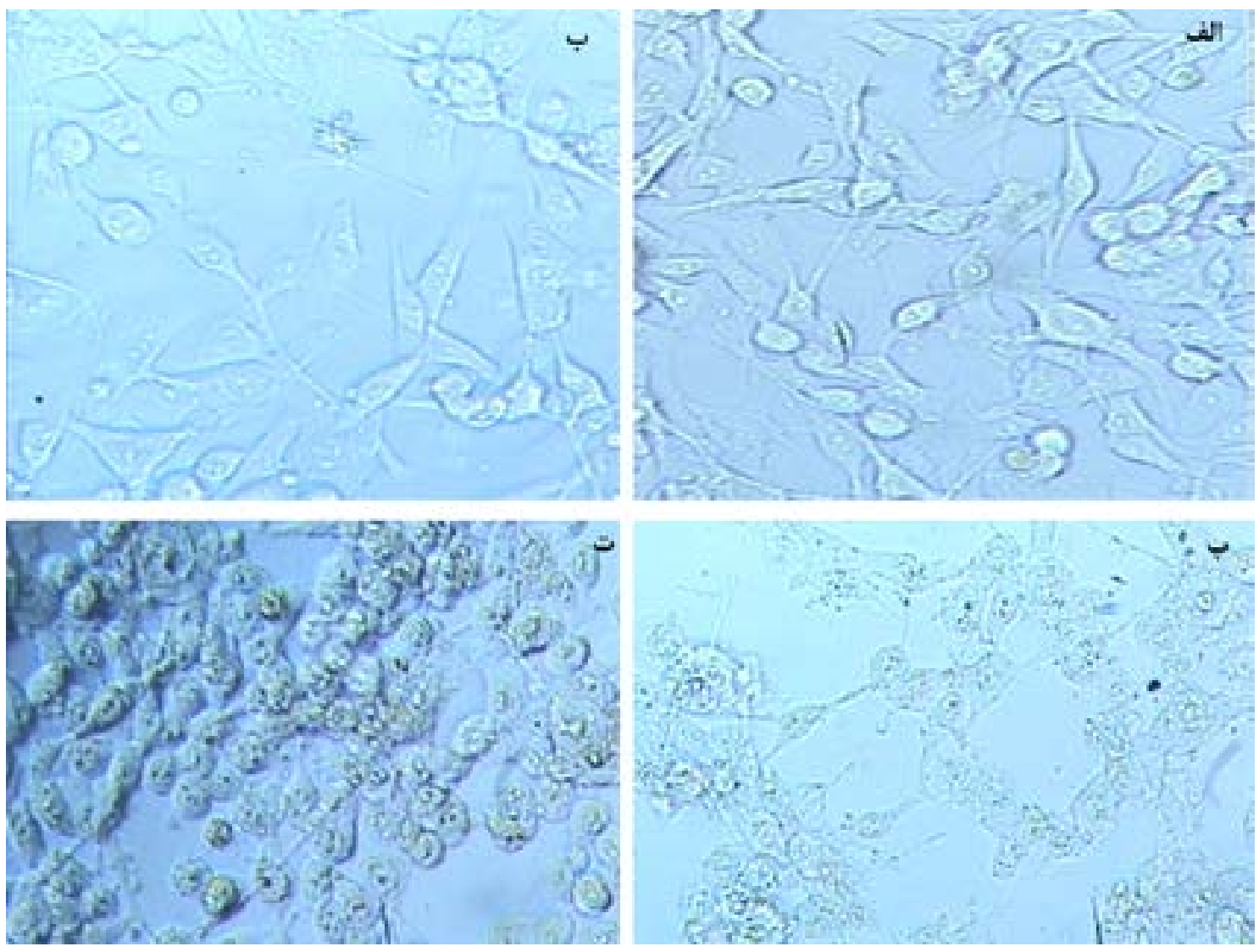

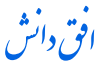
تصوير F. تأثير غلظتهاي مختلف نانوذرات سولفيد روى در سلولهاى فيبروبلاستى استخراجشده از جنين جوجه بس از تيمار 19 ساعته

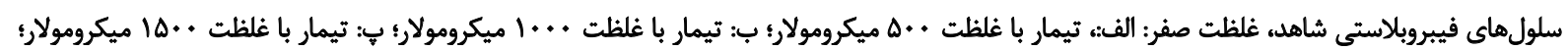
ت: ت سولفيد روى فيرولاني 
روى درصنايع مختلف همجيون داروسازى، به عنوان حامل دارويى

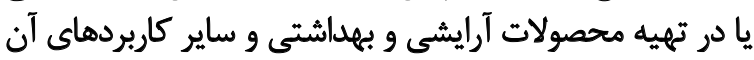

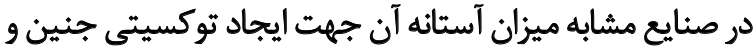
ايجاد ناهنجارى در جنين بايد مورد توجه قرار گيرد.

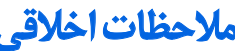

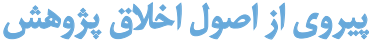

انجام كليه آزمايشها در آزمايشكاه زيستشناسى دانشعاه

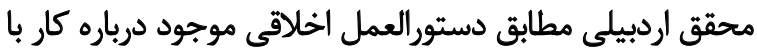

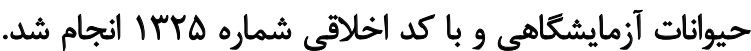

همجينين تلاش شد از تعداد حيوانات كمترى استفاده شود.

$$
\text { مامي مالي }
$$

دانشكاه محقق اردبيلى از اين يُروهش حمايت مالى كرده است.

مشاركت نويسند مان

يزؤهشكر اصلى، روششئاس و نتارنده مقاله: اسداله اسدى

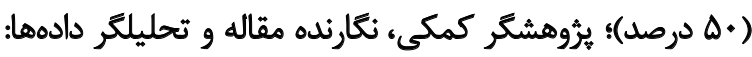

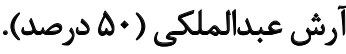

تقارض مثأع

بنابر اظهار نويسئدكان اين مقاله تعارض منافع ندارد.

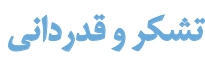

بدينوسيله از معاونت يروهشى دانشكاه محقق اردبيلى تقدير

$$
\text { و تشكر مي شود. }
$$

اثر داروى مثاتوركسات در موشهاى صحرايى نشان داد بيشتر

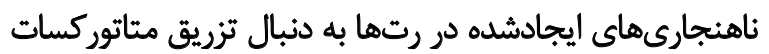

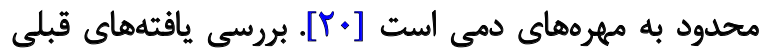

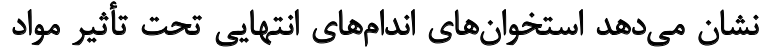

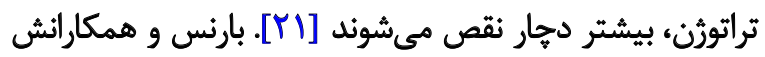

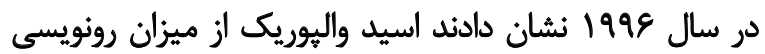

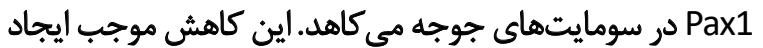

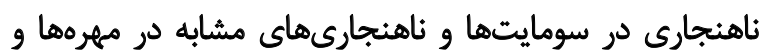

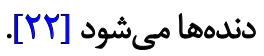

استودنيكا و همكارانش در سال 9 ×. ج اثرات نانوذرات آليار

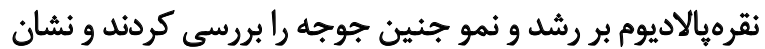

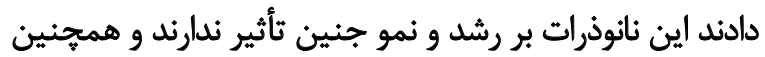

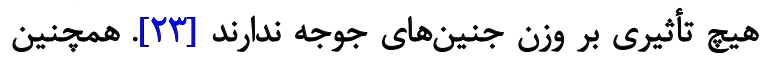

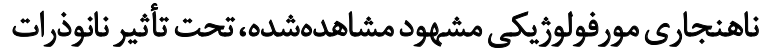

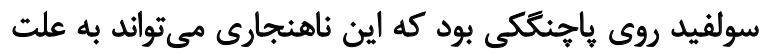

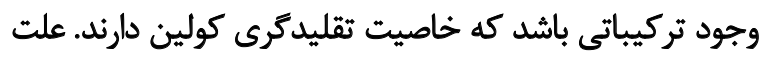

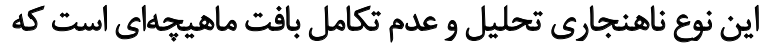

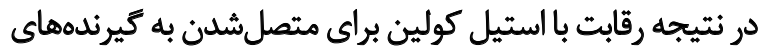

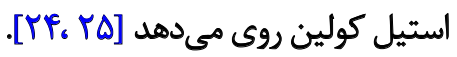

تمام غلظتهاى عامل ناهنجارىزا نمىتواند منشاً توليد

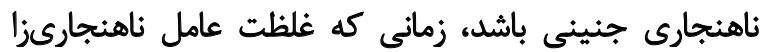

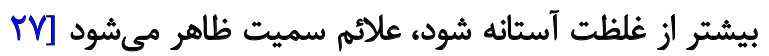

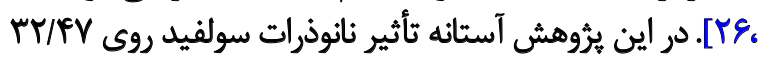

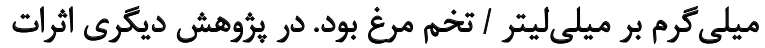

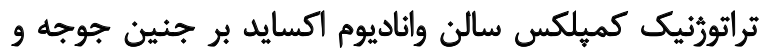

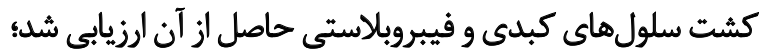

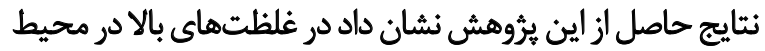

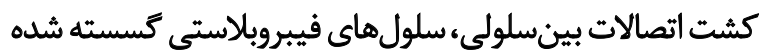

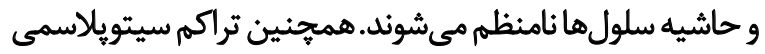
درون سلول افزايش يافته و سلولها واكوئله مي تشود [YA]]. باوانيلاتا و همكارانش اثرات نانوذرات نقره را روى جنين جوجيه

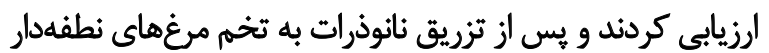

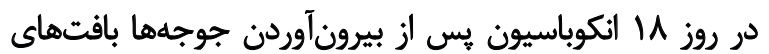

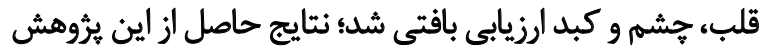

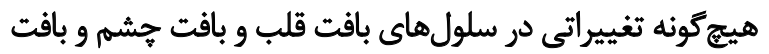

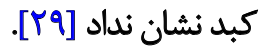

\section{نتيجنئيرى}

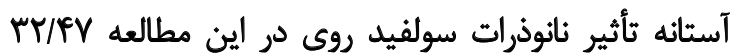

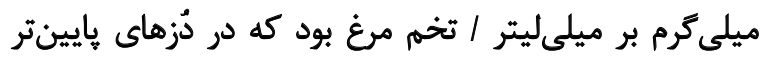

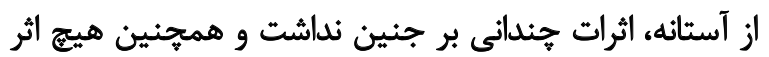

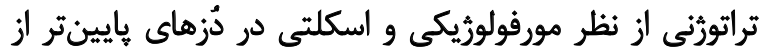

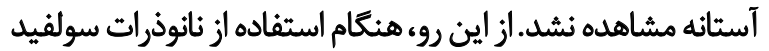




\section{References}

[1] Hullmann A. Measuring and assessing the development of nanotechnology. Scientometrics. 2007; 70(3):739-58. [DOI:10.1007/s11192007-0310-6]

[2] Jones MG, Blonder R, Gardner GE, Albe V, Falvo M, Chevrier J. Nanotechnology and nanoscale science: Educational challenges. International Journal of Science Education. 2013; 35(9):1490-512. [DOI:10.1 080/09500693.2013.771828]

[3] Hardman R. A toxicologic review of quantum dots: Toxicity depends on physicochemical and environmental factors. Environmental Health Perspectives. 2005; 114(2):165-72. [DOI:10.1289/ehp.8284] [PMID] [PMCID]

[4] Wallace WE, Keane MJ, Murray DK, Chisholm WP, Maynard AD, Ong TM. Phospholipid lung surfactant and nanoparticle surface toxicity: Lessons from diesel soots and silicate dusts. In: Maynard AD, Pui DYH, editors. Nanotechnology and Occupational Health. Berlin: Springer; 2006. [DOI:10.1007/978-1-4020-5859-2_4]

[5] Hu H, Wang X, Liu F, Wang J, Xu C. Rapid microwave-assisted synthesis of graphene nanosheets-zinc sulfide nanocomposites: Optical and photocatalytic properties. Synthetic Metals. 2011; 161(5):404-10. [DOI:10.1016/j.synthmet.2010.12.018]

[6] Janzen FJ, Paukstis GL. Environmental sex determination in reptiles: Ecology, evolution, and experimental design. The Quarterly Review of Biology. 1991; 66(2):149-79. [DOI:10.1086/417143]

[7] Abdolmaleki A, Sanginabadi F, Rajabi A, Saberi R. The effect of electromagnetic waves exposure on blood parameters. International Journal of Hematology-Oncology and Stem Cell Research. 2012; 66(2):131-34.

[8] Asadi A, Abdolmaleki A, Najafi F. [Study of teratogenic and cytotoxic effects of bdp18 tri-block copolymer (PLA-pEG2000-pLA) on chicken embryos (Persian)]. Journal of Ardabil University of Medical Sciences. 2013; 13(1):16-23.

[9] Prasek M, Sawosz E, Jaworski S. Influence of nanoparticles of platinum on chicken embryo development and brain morphology. Nanoscale Research Letters. 2013; 8(1):251-9. [DOI:10.1186/1556276X-8-251] [PMID] [PMCID]

[10] Petrovová E, Sedmera D, Misek I, Lesnik F, Luptáková L. Bendiocarbamate toxicity in the chick embryo. Folia Biologica. 2009; 55(2):61-6.

[11] Zahri S, Bezaatpour A, Abdolmaleki A. [Teratogenic and cytotoxic effects of Salen, A current ligand in vanadium complexes (Persian)] Journal of Molecular and Cellular Research. 2014; 27(3):367-76.

[12] Männer J, Seidl W, Heinicke $F$, Hesse $H$. Teratogenic effects of suramin on the chick embryo. Anatomy and Embryology. 2003; 206(3):229-37. [DOI:10.1007/s00429-002-0292-3] [PMID]

[13] Wu X, Li K, Wang H. Facile synthesis of ZnS nanostructured spheres and their photocatalytic properties. Journal of Alloys and Compounds. 2009; 487(1-2):537-44. [DOI:10.1016/j.jallcom.2009.08.010]

[14] Abdolmaleki A, Zahri S. Comparison of toxicity and teratogenic effects of salen and vo-salen on chicken embryo. Drug and Chemical Toxicology. 2016; 39(3):344-9. [DOI:10.3109/01480545.2015.112149 2] [PMID]

[15] Green MC. A rapid method for clearing and staining specimens for the demonstration of bone. The Ohio Journal of Science. 1952; 52(1):31-8.
[16] Bruggeman V, Swennen Q, De Ketelaere B, Onagbesan O, Tona K, Decuypere E. Embryonic exposure to 2, 3, 7, 8-tetrachlorodibenzop-dioxin in chickens: Effects of dose and embryonic stage on hatchability and growth. Comparative Biochemistry and Physiology Part C. 2003; 136(1):17-28. [DOI:10.1016/S1532-0456(03)00168-6]

[17] Roelens SA, Beck V, Maervoet J, Aerts G, Reyns GE, Schepens P, et al. The dioxin-like PCB 77 but not the ortho-substituted PCB 153 interferes with chicken embryo thyroid hormone homeostasis and delays hatching. General and Comparative Endocrinology. 2005; 143(1):1-9. [DOI:10.1016/j.ygcen.2005.02.015] [PMID]

[18] Van der Geyten S, Van den Eynde I, Segers IB, Kühn ER, Darras VM. Differential expression of iodothyronine deiodinases in chicken tissues during the last week of embryonic development. General and Comparative Endocrinology. 2002; 128(1):65-73. [DOI:10.1016/S00166480(02)00065-5]

[19] Panariti A, Miserocchi G, Rivolta I. The effect of nanoparticle uptake on cellular behavior: Disrupting or enabling functions? Nanotechnology, Science and Applications. 2012; 5:87-100. [DOI:10.2147/NSA.S25515] [PMID] [PMCID]

[20] Skalko RG, Gold MP. Teratogenicity of methotrexate in mice. Teratology. 1974; 9(2):159-63. [DOI:10.1002/tera.1420090206] [PMID]

[21] Singh J, Singh S. Skeletal malformations induced by mitomycin C in chick embryos. Acta Orthopaedica. 1976; 47(5):509-14. [DOI:10.3109/17453677608988729] [PMID]

[22] GilbertSF, Epel D. Ecological developmental biology: Integrating epigenetics, medicine, and evolution. Swarthmore: Swarthmore Colledge; 2009.

[23] Studnicka A, Sawosz E, Grodzik M, Chwalibog A, Balcerak M. Influence of nanoparticles of silver/palladium alloy on chicken embryos' development. Annals of Warsaw University of Life Sciences. 2009; 46:237-42.

[24] Forsyth C, Frank A, Watrous B, Bohn A. Effect of coniine on the developing chick embryo. Teratology. 1994; 49(4):306-10. [DOI:10.1002/ tera.1420490410] [PMID]

[25] Landauer W. Cholinomimetic teratogens: Studies with chicken embryos. Teratology. 1975; 12(2):125-45. [DOI:10.1002/tera.1420120206] [PMID]

[26] Magras I, Kotsaki-Kovatsi V, Kovatsis A, Adamidou L. Teratogenic effects of a mixture of scopolamine and hyoscyamine in chick embryos. Veterinary and Human Toxicology Journal. 1993; 35(5):434-5.

[27] Kumar K, Devi K. Teratogenic effects of methyl parathion in developing chick embryos. Veterinary and Human Toxicology Journal. 1992; 34(5):408-10. [PMID]

[28] Abdolmaleki A, Zahri S, Bezaatpour A. [Teratogenic and cytotoxic effects of VO salen complex on chicken embryos, hepatic and fibroblastic- cell cultures (Persian)]. Tehran University of Medical Sciences. 2013; 71(1):7-14.

[29] Muthiah B, Stanley S. In vivo toxicity assessment of silver nanoparticles synthesized from marine sponges (Haliclona implexiformis). Journal of Pharmacy Research. 2010; 3(10):2552-4. 
